\title{
Ga-68 Labeled Somatostatin Analogs Positron Emission Tomography/ Computed Tomography in Gastroenteropancreatic Neuroendocrine Tumors
}

Cigdem Soydal*

Ankara University Medical School, Department of Nuclear Medicine, Ankara, Turkey

Received: December 24, 2014; Accepted: April 04, 2014; Published: April 17, 2014

*Corresponding author: Cigdem Soydal, Ankara University Medical School, Department of Nuclear Medicine, Ankara, Turkey, Tel: +905333137701/+903122406787; E-mail: csoydal@yahoo.com

\begin{abstract}
Neuroendocrine tumors are a heterogeneous group of malignancy and have an increasing incidence. Different diagnostic imaging tools have been performed to evaluate neuroendocrine tumors such as computed tomography and magnetic resonance imaging. However these anatomical methods cannot give information about somatostatin receptor expression, which is extremely important in the management of neuroendocrine tumors. For this reason, somatostatin receptor imaging with radiolabelled somatostatin analogs has an increasing clinical practice. Today, planar scintigraphy or SPECT imaging with Tc-99m or In-111 labeled somatostatin analogs are widely being utilized worldwide. Recently, studies about superiority of positron emission tomography/ computed tomography with Ga-68 labeled somatostatin receptor analogs in the management of neuroendocrine tumors have been reported. The aim of this review is to search the recent literature the role of positron emission tomography/computed tomography with Ga-68 labeled somatostatin receptor analogs in the management of gastroenteropancreatic neuroendocrine tumors.
\end{abstract}

Keywords: Gastroenteropancreatic neuroendocrine tumors; somatostatin receptors; Gallium-68 positron emission tomography

\section{Introduction}

Neuroendocrine tumors (NETs) are characterized by the heterogeneous nature, frequently indolent course and possibility of multiple and variable anatomic sites of the primary tumor. Somatostatin is a cyclic peptide consisted of 14 amino acids and produced by neuroendocrine, immune, and inflammatory cells. The cerebral cortex, the brain stem, the hypothalamus, the pancreas and the gastrointestinal tract are physiological production sites. SSTRs are a family of G-proteincoupled receptors that comprises five subtypes (SSTR1-5) $[1,2]$. Well/moderately differentiated NETs are generally overexpress somatostatin receptors (SSTRs) [3]. Because of the overexpression of SSTRs the radiolabeled somatostatin analogs can be used to localize the primary tumor and its metastasis. The imaging of the SST subtype 2 (SST2) has been developed and has had clinical applications [4]. The majority of NETSs expresses SSTR types 1, 2, 3, and 5 [5]. SSTR2 is the dominant expressed subtype in pancreatic endocrine or carcinoid tumors [6].
Somatostatin receptor imaging is widely used for the diagnosis, staging and restaging of NETs [7]. Firstly, somatostatin receptor scintigraphy with Tc-99m and In-111 labeled somatostatin analogs have been used for the evaluation of NETs [8,9]. After development of Ga-68 labeled somatostatin analogs, in most centers in Europe positron emission tomography (PET) with Ga68 labeled somatostatin analogs have been utilized due to higher resolution of PET imaging. Ga-68 is a positron emitter radio metal with convenient labeling characteristics and it can be easily obtained from Ge-68/Ga-68 generator system [10]. Because of different somatostatin receptor subtype expression of different neuroendocrine tumors, different synthetic somatostatin analogs with different SSTRs subtype affinities have been developed such as DOTA-D-Phe1-Tyr3-octreotide (DOTATOC), DOTA- 1-Nal3octreotide (DOTANOC) and DOTA-D-Phe1-Tyr3-Thr8-octreotide (DOTATATE) (Figure 1). Previous studies have established the efficacy of this Ga-68 labeled DOTA-peptides in the diagnosis of NETs [11-15].

In the comparison with In-111 DTPAOC SPECT, Ga-68 DOTATOC PET is superior in detecting small lesions with low tracer uptake [16]. Superiority of Ga-68 labeled somatostatin
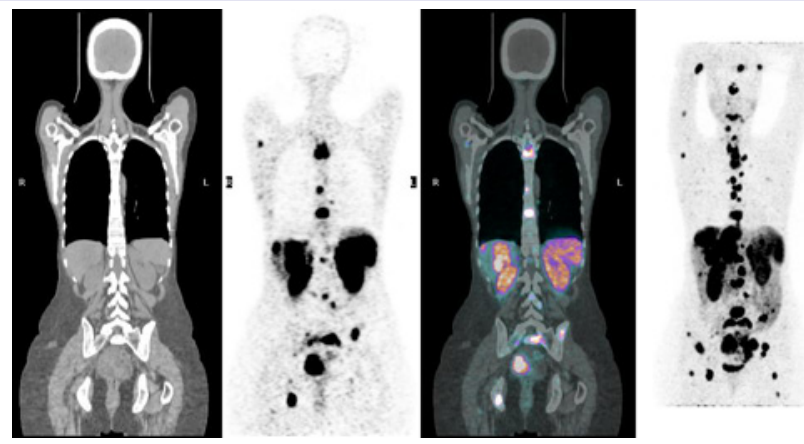

Figure 1: Computed tomography, positron emission tomography, fused and maximum intensity projection images of 56 years old male patient who underwent Ga-68 DOTATATE PET/CT for restaging of gastric neuroendocrine tumor. Intense Ga-68 DOTATATE accumulation was detected in multiple sites in liver, abdominal lymph nodes and bone. 
PET/CT to SPECT imaging is predictable due to higher spatial resolution of PET imaging. In another study included 84 patients with known or suspected NETs, Ga-68 DOTATOC PET had higher detection rate compared with SPECT and diagnostic CT [14] (Figure 2). In addition to higher lesion detection rates in centers that have high patient-flow Ga-68 somatostatin PET/CT imaging might be more economical than In-111 somatostatin SPECT imaging.

Today different synthetic somatostatin analogs are labeled with Ga-68. In a recent study, authors have compared Ga-68 DOTATOC and Ga-68 DOTATATE PET/CT and they have reported that both of them have the same accuracy for the diagnosis of NET lesions. It was also reported although the higher standardized uptake values of Ga-68 DOTATATE, the diagnostic value of PET/ CT with Ga-68 DOTATATE and Ga-68 DOTATOC in the same patients with GEP-NET is almost the same [17] (Figure 3).

\section{Clinical Implications}

\section{Diagnosis, staging and restaging}

Ga-68 somatostatin PET/CT could be utilized for detection of primary tumor and staging of gastroenteropancreatic (GEP) NETs. Ge-68/Ga-68 generator system provides the basis of convenient, easy labeling of somatostatin analogs within 30 minutes. It can give also flexible clinical examination in contrast to cyclotron dependent radiopharmaceuticals. Other advantages of PET imaging are lower radiation doses and short scanning time. The sensitivity and specificity of Ga-68 DOTANOC PET/CT in the detection of primary tumor of GEP NETs have been reported as $78.2 \%$ and $92.5 \%$ respectively. Contrarily the sensitivity in the biochemically confirmed insulinoma patients has been found as low as $18 \%$. Pancreatic NETs have higher uptake than other sites due to their well differentiation and higher SSTR subtype 2 expressions [18]. In a recent study of Gabriel et al. [14] Ga-68 DOTATOC PET/CT has been found superior to In-111 DOTAPAOC in metastasis of GEP-NETs to lung, bones, liver and brain.
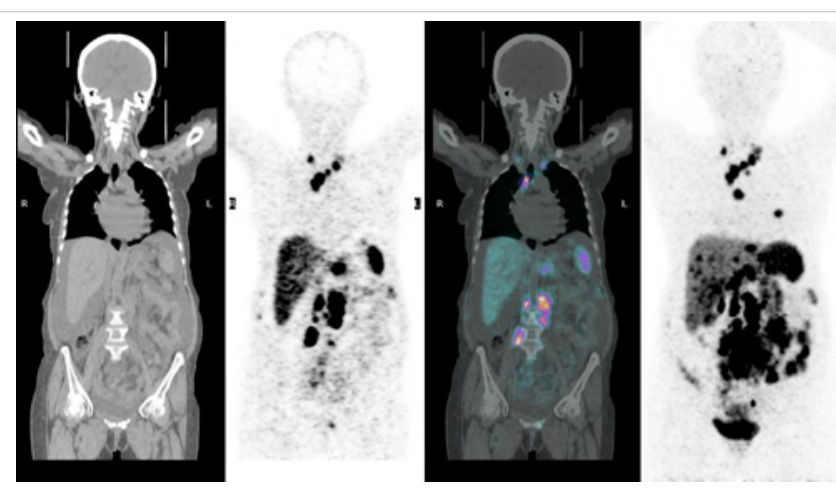

Figure 2: Computed tomography, positron emission tomography, fused and maximum intensity projection images of 58 years old female patient who underwent Ga-68 DOTATATE PET/CT for restaging of pancreatic neuroendocrine tumor. Intense Ga-68 DOTATATE accumulation was seen in liver, abdominal-mediastinal lymph nodes and multiple sites in omental surface.
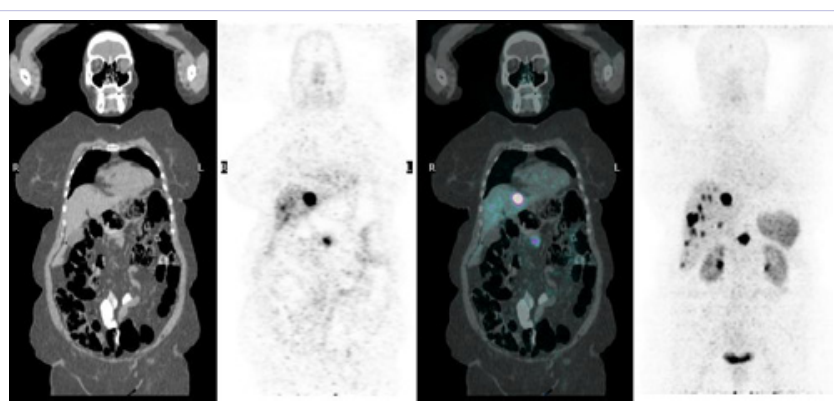

Figure 3: Planar In-111 Octreotide and fused and maximum intensity projection Ga-68 DOTATATE PET/CT images of 43 years old male patient with pancreatic neuroendocrine tumor. Higher resolution of Ga68 DOTATATE PET/CT with intense tracer uptake in pancreatic mass and liver metastasis can be recognized.

In patient based analysis, accuracy of Ga-68 DOTATOC PET/CT was calculated higher than CT and SPECT [14]. Ga-68 DOTATOC $\mathrm{PET} / \mathrm{CT}$ could detect more lesions in lymph nodes, liver and bone. Kowalski et al. have reported that Ga-68 DOTATOC PET was superior to In-111 Octreotide scintigraphy in especially detection of small lesions and tumors that have low density of SSTRs [16]. Ga-68 DOTATATE PET/CT can detect additional lesions in patients who have equivocal lesions in In-111 octreotid scintigraphy and it can change management of patients [19]

In metastatic cases, the sensitivity and specificity of Ga-68 DOTANOC PET/CT are $97.4 \%$ and $100 \%$. It is the best successful modality in the detection of lymph node and bone metastasis. Although liver is the most common metastasis site, there has not been detected superiority of Ga-68 somatostatin PET/CT to CT or MRI [18] (Figure 4).

The relationship between the maximum SUV (SUVmax) in Ga68 DOTATOC PET/CT and the expression of SSTR2 at the level of mRNA has been demonstrated [20]. In another study correlation between SUVmax on Ga-68 somatostatin receptor PET/CT (SSTR PET/CT) and immunohistochemical scores used for the quantitative assessment of the density of subtypes of SSTRs in NET tissue has been proved [21].

\section{Management of patients}

In early stage, surgical resection is the choice of treatment in GEP-NETs. However more patients are diagnosed in the advanced stages of disease and they do not have chance for curative treatments. In this cases different treatment options such as somatostatin analogs, immunological therapy, kinase inhibitors, chemotherapy, peptide receptor radionuclide therapy (PRRNT) can be applied depending to stage of disease, differentiation degree, size and localization. SSTR PET/CT has been utilized in the evaluation of the receptor status before PRRNT or somatostatin analogs [22]. Another indication of Ga-68 SSTR PET/CT is the evaluation of molecular response to therapy by assessing SSTR density in vivo before and after PRRNT [23]. It also helps for detection of recurrence in patients with rising tumor markers. 

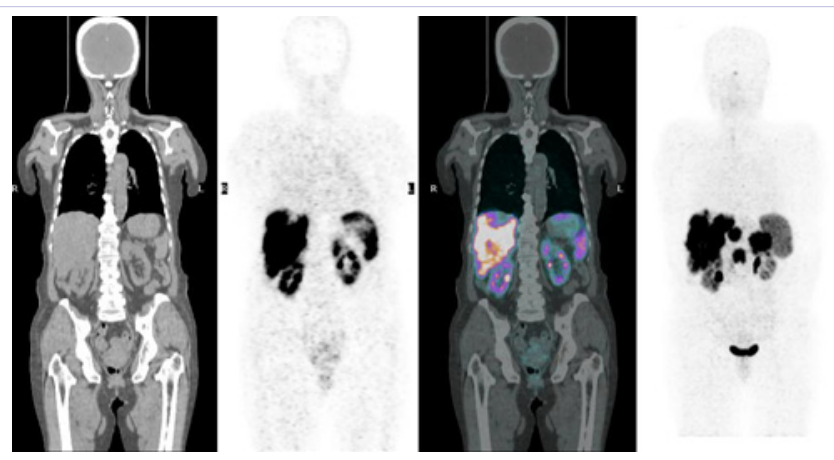

Figure 4: Planar In-111 Octreotide and fused and positron emission tomography Ga-68 DOTATATE PET/CT images of 61 years old male patient with enteric neuroendocrine tumor. Ga-68 DOTATATE accumulation was detected in mesenteric lymph node while In-111 Octreotid planar scintigraphy is normal.

\section{Assessment of treatment response}

Evaluation of treatment response with response evaluation criteria in solid tumors (RECIST) is difficult in well-moderately differentiated GEP-NETs due to slow growing rate. Clinical evaluation is impossible in most nonfunctioning tumors. Moreover, biochemical markers such as chromogranin A or 5-HIAA have poor sensitivity. Ga-68 somatostatin PET/BT could be more effective by giving molecular and morphological information together in the evaluation of treatment response [24].

\section{Conclusion}

Ga-68 somatostatin PET/CT is a successful imaging modality in staging-restaging of well-moderately differentiated gastroenteropancreatic neuroendocrine tumors. In addition to give functional information, it also helps to select good candidates for somatostatin analogs and peptide radionuclide treatment.

\section{References}

1. Patel YC (1999) Somatostatin and its receptor family. Front Neuroendocrinol 20(3): 157-198.

2. Hoyer D, Bell GI, Berelowitz M, Epelbaum J, Feniuk W, et al. (1995) Classification and nomenclature of somatostatin receptors. Trends Pharmacol Sci 16(3): 86-88.

3. Reubi JC, Waser B, Schaer JC, Laissue JA (2001) Somato- statin receptor sst1-sst5 expression in normal and neoplastic human tissues using receptor autoradiography with subtype- selective ligands. Eur J Nucl Med 28(7): 836-846.

4. Reubi JC, Maecke HR (2008) Peptide-based probes for cancer imaging. J Nucl Med 49(11): 1735-1738.

5. Papotti $M$, Bongiovanni $M$, Volante $M$, Allìa $E$, Landolfi $S$, et al. (2002) Expression of somatostatin receptor types 1-5 in 81 cases of gastrointestinal and pancreatic endocrine tumors: a correlative immunohisto- chemical and reverse-transcriptase polymerase chain reaction analysis. Virchows Arch 440(5): 461-475.

6. de Herder WW, Hofland LJ, van der Lely AJ, Lamberts SW (2003) Somatostatin receptors in gastroentero-pancreatic neuroendocrine tumours. Endocr Relat Cancer 10(4): 451-458.
7. Wong KK, Waterfield RT, Marzola MC, Scarsbrook AF, Chowdhury $\mathrm{FU}$, et al. (2012) Contemporary nuclear medicine imaging of neuroendocrine tumours. Clin Radiol 67(11): 1035-1050.

8. Lamberts SW, Reubi JC, Krenning EP (1994) Somatostatin and the concept of peptide receptor scintigraphy in oncology. Semin Oncol 21: $1-5$.

9. Krenning EP, Kwekkeboom DJ, de Jong M, Visser TJ, Reubi JC, et al. (1994) Essentials of pep- tide receptor scintigraphy with emphasis on somatostatin analog octreotide. Semin Oncol 21: 6-14.

10. Rösch F, Baum RP (2011) Generator-based PET radiopharmaceuticals for molecular imaging of tumours: on the way to THERANOSTICS. Dalton Trans 40 (23): 6104-6111.

11.Zhernosekov KP, Filosofov DV, Baum RP, Aschoff P, Bihl H, et al. (2007) Processing of generator-produced $68 \mathrm{Ga}$ for medical application. J Nucl Med 48 (10): 1741-1748.

12. Hofmann M, Maecke H, Börner R, Weckesser E, Schöffski P, et al. (2001) Biokinetics and imaging with the somatostatin receptor PET radioligand (68)Ga-DOTATOC: preliminary data. Eur J Nucl Med 28(12): 1751-1757.

13. Buchmann I, Henze M, Engelbrecht S, Eisenhut M, Runz A, et al. (2007) Comparison of 68Ga-DOTATOC PET and 111In-DTPA-OC (Octreoscan) SPECT in patients with neuroendocrine tumours. Eur J Nucl Med Mol Imaging 34: 1617-1626.

14. Gabriel M, Decristoforo C, Kendler D, Dobrozemsky G, Heute D, et al. (2007) 68Ga-DOTA-Tyr3-octreotide PET in neuroendocrine tumors: comparison with somatostatin receptor scintigraphy and CT. J Nucl Med 48(4): 508-518.

15. Ambrosini V, Campana D, Bodei L, Nanni C, Castellucci P, et al. (2010) 68Ga- DOTA-NOC PET/CT clinical impact in patients with neuroendocrine tumors. J Nucl Med 51(5): 669-673.

16. Kowalski J, Henze M, Schuhmacher J, Mäcke HR, Hofmann M, et al. (2003) Evaluation of positron emission tomography imaging using [68Ga]-DOTA-D Phe(1)-Tyr(3)-Octreotide in comparison to [111In]DTPAOC SPECT. First results in patients with neuroendocrine tumors. Mol Imaging Biol 5(1): 42-48.

17. Poeppel TD, Binse I, Petersenn S, Lahner H, Schott M, et al. (2011) Ga68 DOTATOC versus Ga-68 DOTATATE PET/CT in functional imaging of neuroendocrine tumors. J Nucl Med 52(12): 1864-1870.

18. Baum RP, Rösch F (2013) Theranostics, Gallium-68, and Other Radionuclides. London: Springer 323-324.

19. Srirajaskanthan R, Kayani I, Quigley AM, Soh J, Caplin ME, et al. (2010) The role of Ga-68 DOTATATE PET in patients withneuroendocrine tumors and negative or equivocal findings on In-111 DTPA-octreotide scintigraphy. J Nucl Med 51(6): 875-882.

20. Boy C, Heusner TA, Poeppel TD, Redmann-Bischofs A, Unger N, et al. (2011) (68)Ga-DOTATOC PET/CT and somatostatin receptor (sst1sst5) expression in normal human tissue: correlation of sst2 mRNA and SUV(max). Eur J Nucl Med Mol Imaging 38(7): 1224-1236.

21. Kaemmerer D, Peter L, Lupp A, Schulz S, Sänger J, et al. (2011) Molecular imaging with ${ }^{68} \mathrm{Ga}-\mathrm{SSTR}$ PET/CT and correlation to immunohistochemistry of somatostatin receptors in neuroendocrine tumour. Eur J Nucl Med Mol Imaging 38(9): 1659-1668.

22. Baum RP, PrasadV (2008) PET and PET/CT Imaging of Neuroendocrine Tumors. In: Wahl R, ed. Principles and Practice of PET and PET/CT (2nd ed) Philadelphia: Lippincott Williams \& Wilkins: 411-437. 
23. Baum RP, Prasad V, Hommann M, Hörsch D (2008) Receptor PET/CT imaging of neuroendocrine tumors. Recent Results in Cancer Research 170: $225-242$.
24. Baum RP, Kulkarni HR, Carreras C (2012) Peptides and receptors in image-guided therapy: theranostics for neuroendocrine neoplasms. Semin Nucl Med 42(3): 190-207. 